\section{AB0886 DOES RADIO-ANATOMIC FEATURE OF HIP ARTHRITIS IN SPONDYLOARTHRITIS PREDICT FUNCTIONAL IMPAIRMENT?}

W. hamdi, R. Ben Saad, H. Riahi, K. Maatallah, D. Kaffel, T. Bouaziz, L. Ledab, M. Kchir. institut national de Mohamed El Kassab, tunis, Tunisia

Background: Axial spondyloarthritis (AS) is a chronic inflammatory disease that primarily affects the axial skeleton. Hip involvement is common in AS patient. The reported prevalence of clinical hip involvement in AS ranges from $24 \%$ to $36 \%$ and prevalence of radiographic hip arthritis ranges from $9 \%$ to $22 \% .{ }^{1,2}$ Several radio-anatomic feature are described with often significant clinical impact.

Objectives: To assess for an association between the radio-anatomic feature of hip arthritis and its clinical impact in patients with AS.

Methods: Cross-sectional study including patients with AS according to the ${ }^{\text {ASAS }}$ criteria of 2009 with hip arthritis. All patients had pelvic X-rays. This one allowed to distribute the patients according to five radio-anatomic feature of the hip arthritis: destructive, protrusive, ankylosing, mimicking osteoarthritis and early hip arthritis. To evaluate the functional impairement, two clinical indices were calculated: Lequesne index and Intermalleolar distance between the two medial malleoli in centimetre. The comparison of qualitative variables was performed with the Chi square test and the comparison of qualitative variable and quantitative ones was performed with the Student's test. The significance level was set at 0.05 .

Results: One hundred patients were included with 176 hips arthritis. The delay between onset of AS and hip arthritis was on average 5.6 years [0-34]. The distribution of coxitis according to the radio-anatomic feature was as following: destructive (110 cases), protrusive (10 cases), mimicking osteoarthritis (29 cases), ankylosing ( 7 cases) and early hip arthritis (20 cases).

According to the Larsen classification for destructive hip arthritis: a grade 4 was found in 41 cases, a grade 2 in 33 cases, a grade 3 in 33 cases and a grade 1 in 3 cases.

The destructive form had the most important functional impairment with the highest Lequesne index in comparison with other forms $(13,05$ vs $9,7, p=0,000)$ and the smallest intermalleolar distance $(74,40$ vs $87,29, p=0,010)$.

The early hip arthritis and protrusive forms had the least important functional impact in with the following scores: Lequesne index $(8,83$ vs $12,29, p=0,015)$ and $(10,78$ vs $11,96, p=0,000)$ and the intermalleolar distance $(76,36$ vs 65,00 $p=0,015)$ and $(105,56$ vs $70,22, p=0,002)$ respectively.

Scores were comparables for osteoarthritis and ankylosing forms.

Conclusions: Our study showed that radio-anatomic feature of hip arthritis could influence functional impairment. Distinguishing these forms may allow the rheumatologist to select the hip arthritis that deserves more attention because of the highest risk of functional impairement.

\section{REFERENCES:}

[1] Jeong H, Eun YH, Kim IY, Kim H, Lee J, Koh EM, et al. Characteristics of hip involvement in patients with ankylosing spondylitis in Korea. Korean J Intern Med. 2017;32(1):158-64.

[2] Zhao J, Zheng W, Zhang C, Li J, Liu D, Xu W. Radiographic hip involvement in ankylosing spondylitis: factors associated with severe hip diseases. J Rheumatol. 2015;42(1):106-10.

Disclosure of Interest: None declared

DOI: 10.1136/annrheumdis-2018-eular.5912

\section{AB0887 JIA PATIENTS WITH HIGH DISEASE ACTIVITY HAVE INCREASED ACTIVITY OF BOTH THE IDO AND GTP-CH1 PATHWAY, BUT DECREASED BH4 EFFICACY: CONSEQUENCES FOR FATIGUE AND WELL-BEING}

S. Korte ${ }^{1}$, E. Albers ${ }^{1}$, W. van den Bogaard ${ }^{2}$, G. Korte-Bouws ${ }^{2}$, S. de Roock ${ }^{3}$, S. Vastert'. ${ }^{3}$ Dept of Pharmacology; ${ }^{2}$ Onderwijscentrum Farmacie, Utrecht University, ${ }^{3}$ Paediatric Rheumatology, Wilhelmina Children's Hospital, University Medical Center, UTRECHT, Netherlands

Background: Juvenile idiopathic arthritis (JIA) patients suffer from an inflammatory condition, resulting in painful joints. In addition, JIA patients often report symptoms of sickness behaviour, including depressive symptoms and fatigue. Recent animal studies suggest that proinflammatory cytokines produce sickness behaviour by increasing the activity of indoleamine-2,3-dioxygenase (IDO) and guanosinetriphosphate-cyclohydrolase-1 (GTP-CH1). Here it is hypothesised that inflammation in JIA patients affects the enzymatic activity of IDO and GTP-CH1 and the co-factor $\mathrm{BH} 4$. These compounds play a crucial role in the metabolism of the neurotransmitters serotonin and dopamine.

Objectives: The aim of our study was to reveal whether inflammation affects BH4, IDO and GTP-CH1 (enzymatic) activity in JIA patients.

Methods: Serum samples were collected of twenty-four JIA patients. In these samples, the concentrations of tryptophan, kynurenine, tyrosine, neopterin and phenylalanine were measured. An HPLC method with electrochemical detection was developed to quantify tryptophan, kynurenine and tyrosine. Neopterin and phenylalanine were quantified by ELISA. Kyn/trp ratio was measured as an index of IDO activity, while Phen/Tyr ratio was measured as an index of $\mathrm{BH} 4$ activity. Neopterin concentrations were used as an indirect measure of GTP-CH1 activity. Results: JIA patients with high disease activity showed higher levels of both neopterin and kynurenine, and a higher ratio of both Kyn/Trp and Phen/Tyr and lower tryptophan levels than clinically inactive patients.

Conclusions: Altogether, these data support our hypothesis that inflammation increases the enzymatic activity of both IDO and GTP-CH1 and decreases the efficacy of the co-factor $\mathrm{BH} 4$. Further animal studies are needed to investigate whether inflammation-induced changes in these enzymatic pathways and co-factor lower the levels of the brain neurotransmitters dopamine and serotonin, and consequently produce sickness behaviour and fatigue.

Acknowledgements: This study was financially supported by the focus area "Future Food Utrecht" of Utrecht University, The Netherlands.

Disclosure of Interest: None declared

DOI: 10.1136/annrheumdis-2018-eular.3739

\section{AB0888 PLATELET COUNT IN ANKYLOSING SPONDYLITIS: CAN IT SHOW THE DISEASE ACTIVITY ?}

S. Rahmouni, M. Slouma, R. Dhahri, L. Metoui, N. Boussetta, N. Gueddiche,

F. Laajili, I. Gharsallah, B. Louzir. internal medicine, Military hospital of instruction of Tunis, TUNIS, Tunisia

Background: C-reactive protein (CRP) and erythrocyte sedimentation rate (ESR) are the main inflammatory biomarkers used in assessment of disease activity in numerous inflammatory diseases such as ankylosing spondylitis (SA). In recent years, it has been shown that Platelet count (PC) provided additional information on inflammatory status.

Objectives: The present study aimed to investigate the correlation between PC and clinical activity indices assessed by Bath ankylosing spondylitis disease activity index (BASDAI)

Methods: The study included 68 patients who fulfilled Assessment of Spondyloarthritis International Society Criteria. A cell blood count, including platelet count, $\operatorname{ESR}(\mathrm{mm} / \mathrm{h}), \operatorname{CRP}(\mathrm{mg} / \mathrm{L})$ and albumin $(\mathrm{g} / \mathrm{L})$, was obtained for each patient.

Disease activity measures at the time of blood sampling were obtained using the BASDAI. Statistical Package for Social Sciences (SPSS) was used for analysis.

Results: Of the 68 patients, $73.5 \%$ were male (Sex Ratio: 2.7 ). The mean age was $36 \pm 12.9$ years. The mean disease duration was 40 months [0-192]. The average age of the onset of the disease was $36.6 \pm 11.9$ years. No patient had ongoing infection at time of study. The mean BASDAI score was 5.1 [1.55-9.2]. Forty-one $\%$ of our patients $(n=28)$ had active AS (BASDAI $>4$ )

The mean ESR, CRP, albumin and PC were $39.4(\mathrm{~mm} / \mathrm{h}), 34.1(\mathrm{mg} / \mathrm{dL}), 39,32 \mathrm{~g} / \mathrm{L}$ and $311\left(10^{3} / \mathrm{mL}\right)$, respectively.

There was a positive correlation between platelet count and following biomarkers: $\operatorname{ESR}(r:-0.481 ; p: 0.000), \operatorname{CRP}(r: 0.417 ; p: 0.000)$ and albumin levels (r: -0.556 $\mathrm{p}: 0.000)$. A negative correlation between and Haemoglobin $(\mathrm{Hg})$ was noted $(r$ : $-0.643 ; p: 0.000)$.

There was no significant difference in laboratory parameters (ESR, CRP, albumin, $\mathrm{PC}, \mathrm{Hg}$ ) between patients with active AS (BASDAl $>4$ ) and those with inactive AS (BASDI <4) patients.

Conclusions: Although acute phase reactant such as ESR, CRP and PC mirror disease activity in $A S,{ }^{1}$ their values were not associated with disease activity assessed by BASDAI in our study. However, new prospective studies including larger study groups are required to verify the findings of the present study.

\section{REFERENCE:}

[1] Ruof J, Stucki G. Validity aspects of erythrocyte sedimentation rate and Creactive protein in ankylosing spondylitis: a literature review. J Rheumatol 1999;26:966e70

Disclosure of Interest: None declared

DOI: 10.1136/annrheumdis-2018-eular.5646 